\title{
A delicate balance: Bleeding versus thrombosis?
}

\author{
Robert B. Hawkins, MD, MSc, and J. Hunter Mehaffey, MD, MSc
}

\author{
From the Division of Thoracic and Cardiovascular Surgery, University of Virginia, Charlottesville, Va. \\ Disclosures: Authors have nothing to disclose with regard to commercial support. \\ Received for publication Oct 15, 2018; accepted for publication Oct 17, 2018; available ahead of print Nov 16, \\ 2018. \\ Address for reprints: Robert B. Hawkins, MD, MSc, University of Virginia, Department of Thoracic and Cardio- \\ vascular Surgery, PO Box 800679, Charlottesville, VA, 22908-0709 (E-mail: rbh6x@ virginia.edu). \\ J Thorac Cardiovasc Surg 2019;157:653-4 \\ $0022-5223 / \$ 36.00$ \\ Copyright (C) 2018 by The American Association for Thoracic Surgery \\ https://doi.org/10.1016/j.jtcvs.2018.10.063
}

The mortality and graft patency benefits of early postoperative aspirin after coronary artery bypass grafting (CABG) are clear, and discharge with an antiplatelet agent is now a quality metric. ${ }^{1}$ Somehow, however, preoperative aspirin remains a controversial topic. There is wide variation in practice because of the difficulty in balancing bleeding with thrombosis. On the one hand, a not insignificant number of vein grafts thrombose even before the patient leaves the hospital. Although the effect of asymptomatic graft thrombosis remains unclear, many patients ultimately require interventions for graft patency or have suffer cardiovascular complications. On the other hand, preoperative aspirin is known to slightly increase bleeding. This is notable, given recent work in this Journal exploring just how damaging transfusions can be. ${ }^{2}$

Alternatively, we have known for some time about the benefit in bleeding reduction provided by antifibrinolytic therapy. ${ }^{3}$ In the trial by Myles and colleagues ${ }^{4}$ in this issue of the Journal, tranexamic acid use was associated with fewer transfusions and reoperations for bleeding. ${ }^{4}$ At 1 year, transfusion continued to have a sizeable effect on death or disability (relative risk [RR], 3.91). With around 3 decades of experience with tranexamic acid and aminocaproic acid, the thrombosis concerns of these agents have not been borne out. What the study of Myles and colleagues ${ }^{4}$ adds after all this time is the factorial $2 \times 2$ design across 31 centers and 7 countries. This broad experience should be widely applicable to most cardiac surgeons. What we find is that the combination of preoperative aspirin with tranexamic acid is likely the best option to balance bleeding and thrombosis. The test for interaction between tranexamic acid and aspirin nearly reached statistical significance $(P=.07)$, which is notable when you consider the RR of 1-year death or disability for tranexamic acid with aspirin trends toward protective (RR, 0.7; $P=.052$ ), whereas tranexamic acid without aspirin trends toward harmful (RR. 1.21; $P=.073$ ).

Myles and colleagues ${ }^{4}$ provide minimal evidence, however, for the trend in reduction of 1-year major adverse cardiac events. We posit that the reduction in transfusions is a

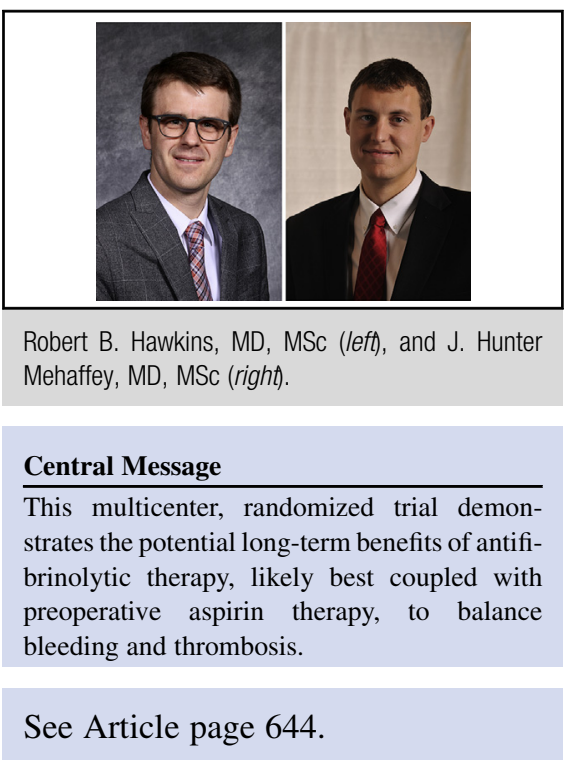

significant contributor. Transfusions are thought to have long-term effects, with some studies finding differences even in 5-year mortality. ${ }^{5}$ Similarly, reoperation may affect long-term survival or may indicate worse bleeding with greater burden of transfusion. Myles and colleagues ${ }^{4}$ are to be congratulated on their rigorous work and their finding of the potential benefit and interaction of tranexamic acid and aspirin. They should, however, delve further into the mechanism of the long-term outcomes and the possible relationship with transfusions that their data suggest.

This study is a reminder for programs to take a close look at their antiplatelet and antifibrinolytic protocols. With clear evidence for high-dose postoperative aspirin, surgeons should be selecting patients who should not receive this therapy within 6 hours, as opposed to the other way around. Surgeons should discuss their preference regarding preoperative aspirin, with this trial supporting its use when in combination with antifibrinolytic agents. Finally, programs should discuss which intraoperative antifibrinolytic agent they wish to use. Most appear equally efficacious, although tranexamic acid may have a dose-dependent increased seizure risk and higher cost compared with other agents, such as aminocaproic acid. This study provides highquality evidence to help balance the intertwined risk of bleeding and thrombosis in cardiac surgery.

\section{References}

1. Mangano DT, Multicenter Study of Perioperative Ischemia Research Group. Aspirin and mortality from coronary bypass surgery. N Engl J Med. 2002;347: 1309-17. 
2. LaPar DJ, Hawkins RB, McMurry TL, Isbell JM, Rich JB, Speir AM, et al. Preoperative anemia versus blood transfusion: which is the culprit for worse outcomes in cardiac surgery? J Thorac Cardiovasc Surg. 2018;156:66-74.e62.

3. Horrow JC, Hlavacek J, Strong MD, Collier W, Brodsky I, Goldman SM, et al. Prophylactic tranexamic acid decreases bleeding after cardiac operations. J Thorac Cardiovasc Surg. 1990;99:70-4.
4. Myles PS, Smith JA, Kasza J, Silbert B, Jayarajah M, Painter T, et al. Tranexamic acid in coronary artery surgery: one-year results of the Aspirin and Tranexamic Acid for Coronary Artery Surgery (ATACAS) trial. J Thorac Cardiovasc Surg. 2019;157:644-52.e9.

5. Bhaskar B, Dulhunty J, Mullany DV, Fraser JF. Impact of blood product transfusion on short and long-term survival after cardiac surgery: more evidence. Ann Thorac Surg. 2012;94:460-7. 